\title{
Analytical Model for Two-Dimensional Electron Gas Charge Density in Recessed-Gate GaN High-Electron-Mobility Transistors
}

\author{
Samaneh Sharbati ${ }^{1} \cdot$ Iman Gharibshahian $^{2} \cdot$ Thomas Ebel $^{1} \cdot$ Ali A. Orouji ${ }^{2} \cdot$ Wulf-Toke Franke $^{1}$
}

Received: 9 November 2020 / Accepted: 24 February 2021 / Published online: 20 April 2021

(c) The Author(s) 2021

\begin{abstract}
A physics-based analytical model for GaN high-electron-mobility transistors (HEMTs) with non-recessed- and recessed-gate structure is presented. Based on this model, the two-dimensional electron gas density (2DEG) and thereby the on-state resistance and breakdown voltage can be controlled by varying the barrier layer thickness and $\mathrm{Al}$ mole fraction in non-recessed depletion-mode GaN HEMTs. The analytical model indicates that the 2DEG charge density in the channel increases from $2.4 \times 10^{12} \mathrm{~cm}^{-2}$ to $1.8 \times 10^{13} \mathrm{~cm}^{-2}$ when increasing the Al mole fraction from $x=0.1$ to 0.4 for an experimental non-recessedgate GaN HEMT. In the recessed-gate GaN HEMT, in addition to these parameters, the recess height can also control the 2DEG to achieve high-performance power electronic devices. The model also calculates the critical recess height for which a normally-ON GaN switch becomes normally-OFF. This model shows good agreement with reported experimental results and promises to become a useful tool for advanced design of GaN HEMTS.
\end{abstract}

Keywords GaN high-electron-mobility transistor $(\mathrm{HEMT}) \cdot$ two-dimensional electron gas (2DEG) · recessed gate $\cdot$ recess height

\section{Introduction}

GaN high-electron-mobility transistors (HEMTs) have shown great potential for use in high-power and high-frequency applications due to their wide bandgap and high electron mobility. ${ }^{1,2}$ The defining feature of this device technology is the presence of a high-density two-dimensional electron gas (2DEG) at the $\mathrm{AlGaN}-\mathrm{GaN}$ interface

Samaneh Sharbati

sharbati@sdu.dk

Iman Gharibshahian

i.gharibshahian@semnan.ac.ir

Thomas Ebel

ebel@sdu.dk

Ali A. Orouji

aliaorouji@semnan.ac.ir

Wulf-Toke Franke

franke@sdu.dk

1 Department of Mechanical and Electrical Engineering, Centre for Industrial Electronics, University of Southern Denmark, 6400 Sønderborg, Denmark

2 Department of Electrical and Computer Engineering, Semnan University, Semnan 3513119111, Iran due to the strong spontaneous and piezoelectric polarization. However, this high 2DEG density leads to GaN HEMTs that are normally-ON (depletion-mode) switches, which is not suitable for power electronic applications because of safety and system cost concerns. Therefore, the carriers in the 2DEG beneath the gate should be depleted to achieve normally-OFF (enhancement (E)-mode) transistors. Moreover, E-mode GaN HEMTs have attracted much attention for use in power switching applications, owing to their high breakdown voltage and low on-resistance. ${ }^{3-9}$

Several techniques have been used to design E-mode GaN devices, all of which incorporate technologies that empty the 2DEG channel underneath the gate at zero gate bias. The recessed-gate structure is one of the approaches for designing E-mode GaN HEMTs for use in power electronic applications. ${ }^{10}$

Understanding the physical mechanisms behind the formation of the 2DEG is crucial to model the 2DEG charge density under the gate and to device design. ${ }^{11}$ Experimental research has shown that the presence of donor states at the AlGaN surface is the main source of the electrons in the 2DEG. ${ }^{12,13}$ For non-recessed-gate GaN HEMT structures, Gordon et al. ${ }^{14}$ showed that the $2 \mathrm{DEG}$ density varies with the barrier layer thickness. It was subsequently shown 
experimentally as well as by numerical simulations that the $\mathrm{Al}$ mole fraction in the $\mathrm{AlGaN}$ has a significant impact on the 2DEG density due to its effect on the piezoelectric and spontaneous polarization.

In this paper, an analytical model for the 2DEG charge density of GaN HEMTs is presented and verified by a complete match with experimental data. Moreover, an analytical model for the recessed-gate GaN HEMT structure is also provided. In addition to the AlGaN barrier layer thickness $(d)$ and the Al mole fraction $(x)$ affecting the density of the 2DEG, the recess height $(h)$ also influences the density of the 2DEG for recessed-gate GaN HEMTs. To design highly efficient and reliable E-mode HEMT structures, it is essential to understand the dependence of the 2DEG density on these physical characteristics.

\section{Simulation of Non-recessed and Recessed-Gate GaN HEMTs and Comparison with Experimental Data}

Figure 1 shows a schematic of an experimental GaN HEMT with the recessed-gate structure. ${ }^{10}$ The epitaxial structure consists of a 15.6-nm-thick AlGaN barrier layer with $\mathrm{Al}$ mole fraction $x=0.27$ on a $2-\mu \mathrm{m}$-thick GaN buffer layer grown on a sapphire substrate. The devices are fabricated with Ti/Al/Ni/Au (20/120/20/70 nm) ohmic metallization at the source and drain. According to the gate, a 3-nm barrier is etched down by neutral-beam technology to recess the gate.

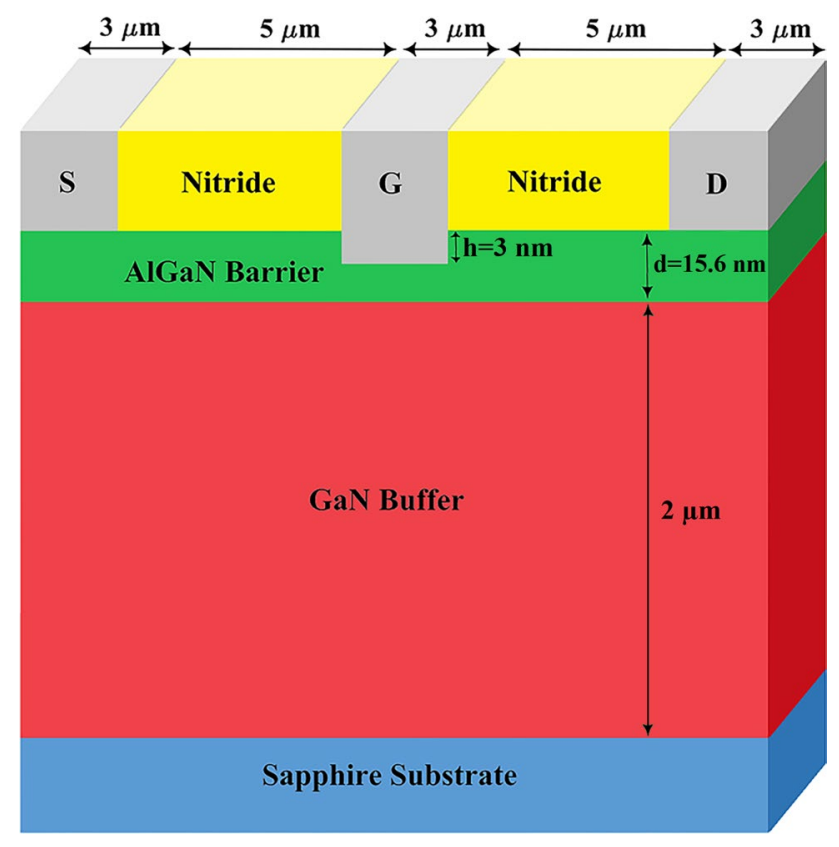

Fig. 1 Schematic of a recessed-gate GaN HEMT structure.
After that, Ni/Au is deposited to form the gate electrode. The detailed fabrication process is explained in Ref. 15.

To achieve precise control over the recess etch rate, neutral-beam etching (NBE) is carried out, and the reduced $\mathrm{AlGaN}$ barrier is formed by low-damage recess etching using the $\mathrm{Cl}_{2}$ plasma process. A radiofrequency (RF) power supply can accurately control the etching rate of AlGaN in GaN-based HEMTs. ${ }^{16,17}$ In this structure with 3-nm recess height, the recess etch rate in the NBE gate recess process is controlled to $5 \mathrm{~nm} / \mathrm{min}$, corresponding to RF bias power. ${ }^{15}$

The electrical characteristics of both devices (with recessed and non-recessed gate) are extracted by solving a two-dimensional (2D) drift-diffusion model. The activated models are the self-heating model to enable heat flow simulation, the $n_{\mathrm{i}}$ Fermi model to include the effects of Fermi statistics into the calculation of the intrinsic concentration in expressions for the Shockley-Read-Hall (SRH) recombination, the polarization model for automatic calculation of the interface charge due to spontaneous and piezoelectric polarization, the strain model for automatic calculation of the strain from the lattice mismatch, and the impact ionization model. Furthermore, the charge at the $\mathrm{AlGaN}-\mathrm{GaN}$ interface is fixed and the donor and acceptor traps at the $\mathrm{GaN}$ buffer are activated. Moreover, it is considered that there are no trapped charges at the interfaces between the GaN buffer layer and sapphire substrate. Table I lists the parameter values adopted in the simulations.

To validate this work, the simulation results and experimental data ${ }^{10}$ for the $I_{\mathrm{D}}-V_{\mathrm{G}}$ transfer and $I_{\mathrm{D}}-V_{\mathrm{D}}$ characteristics for both structures, viz. the non-recessed and recessedgate GaN HEMTs, are compared. The match obtained between the simulation results and experimental data confirms the accuracy of the model for use in further research.

Figure $2 \mathrm{a}$ and $\mathrm{b}$ show the simulated and measured ${ }^{10}$ transfer characteristics of the GaN HEMT for the non-recessed design and with a 3-nm gate recess, respectively. Figure $3 \mathrm{a}$ and $b$ show a perfect match of the simulation of the directcurrent (DC) characteristics with experimental results ${ }^{10}$ for different gate voltages, further verifying the accuracy of the physical models used for the GaN HEMTs. The calculated and measured data for both the non-recessed and recessedgate GaN HEMT are presented in Table II. The maximum drain current $\left(I_{\mathrm{D}-\max }\right)$ is calculated to be around $444 \mathrm{~mA} /$ $\mathrm{mm}$ and $372 \mathrm{~mA} / \mathrm{mm}$ for the non-recessed and recessed-gate structure, respectively, at $V_{\mathrm{G}}=+2 \mathrm{~V}$, while $V_{\mathrm{th}}$ for the nonrecessed gate structure is obtained as $-2.3 \mathrm{~V}$ versus $-1.6 \mathrm{~V}$ for the recessed-gate structure. For the 3-nm gate recess, $V_{\text {th }}$ shifts significantly, while there is a minor reduction in $I_{\mathrm{D}-\max }$ and $g_{\mathrm{m}}$.

The reduction in the current density for gate-recessed devices has been attributed to the diminution of the polarization sheet charge. Moreover, the 2DEG charge density $\left(n_{\mathrm{s}}\right)$ is calculated while there is no voltage. The overlapping 
Table I Material parameters used in both GaN HEMT devices simulations; data from Refs. 18-22.

\begin{tabular}{lcccc}
\hline Parameter & Description & $\mathrm{GaN}$ & $\mathrm{Al}_{0.27} \mathrm{Ga}_{0.73} \mathrm{~N}$ & Units \\
\hline$E_{\mathrm{g}}$ & Bandgap at 300 K & 3.42 & 3.95 & $\mathrm{eV}$ \\
$\chi$ & Electron affinity & 4.31 & 4.04 & $\mathrm{eV}$ \\
$A_{n}{ }^{*}$ & Richardson constant for electrons & 22.4 & 25.3 & $\mathrm{~A} / \mathrm{cm}^{2} \mathrm{~K}^{2}$ \\
$A_{\mathrm{p}}{ }^{*}$ & Richardson constant for holes & 61 & 108 & $\mathrm{~A} / \mathrm{cm}^{2} \mathrm{~K}^{2}$ \\
$N_{\mathrm{c}}$ & Conduction-band effective density of states at $300 \mathrm{~K}$ & $2.02 \times 10^{18}$ & $2.42 \times 10^{18}$ & $\mathrm{~cm}^{-3}$ \\
$N_{\mathrm{v}}$ & Valence-band effective density of states at $300 \mathrm{~K}$ & $9.08 \times 10^{18}$ & $2.15 \times 10^{19}$ & $\mathrm{~cm}^{3}$ \\
$\varepsilon_{\mathrm{r}}$ & Relative permittivity & 10.4 & 10.32 & \\
$\mu$ & Low-field electron mobility & 1460 & 306 & $\mathrm{~cm}^{2} / \mathrm{Vs}$ \\
$v_{\text {sat }, n}$ & Saturation velocity of electrons & $1.91 \times 10^{7}$ & $1.12 \times 10^{7}$ & $\mathrm{~cm} / \mathrm{s}$ \\
$v_{\text {sat }, p}$ & Saturation velocity of holes & $1 \times 10^{6}$ & $1 \times 10^{6}$ & $\mathrm{~cm} / \mathrm{s}$ \\
\hline
\end{tabular}
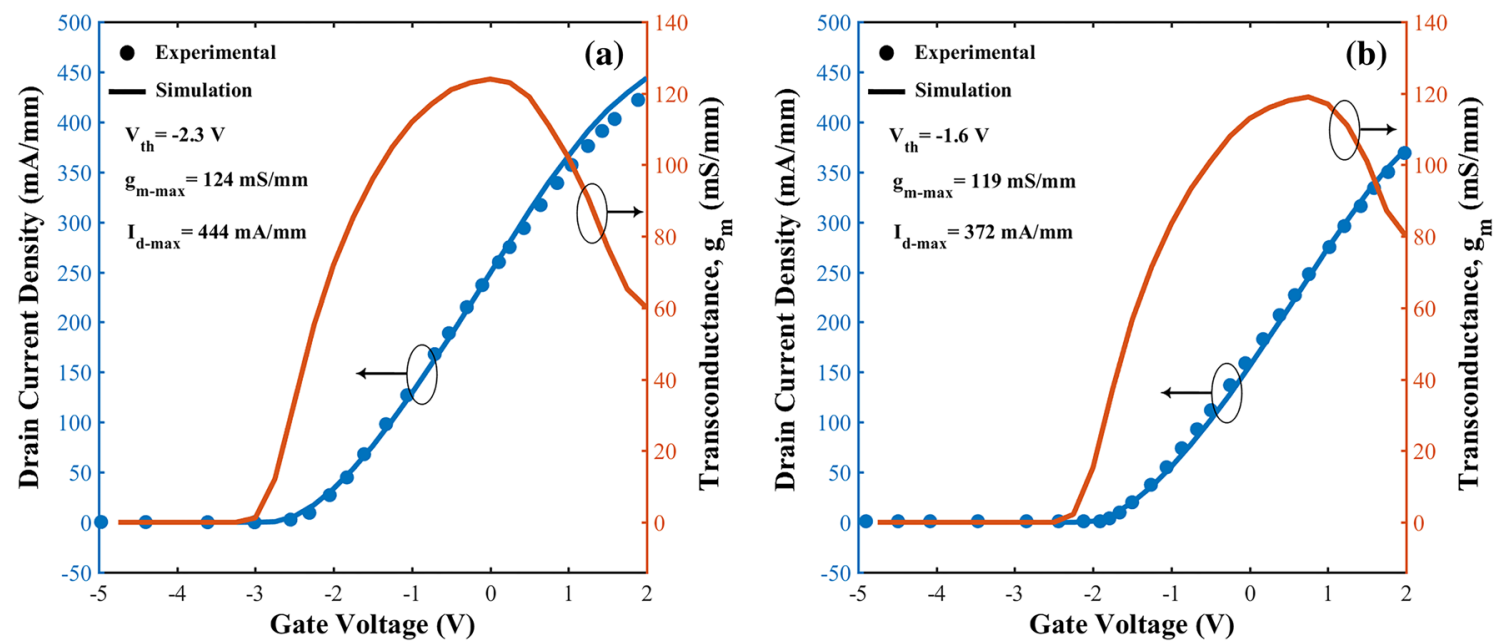

Fig. 2 Transfer characteristics of (a) non-recessed and (b) 3-nm recessed-gate GaN HEMT with $V_{\mathrm{D}}$ of $5 \mathrm{~V}$, AlGaN thickness $(d)$ of $15.6 \mathrm{~nm}$, and Al mole fraction $(x)$ of 0.27 . (Experimental data from Ref. 10).
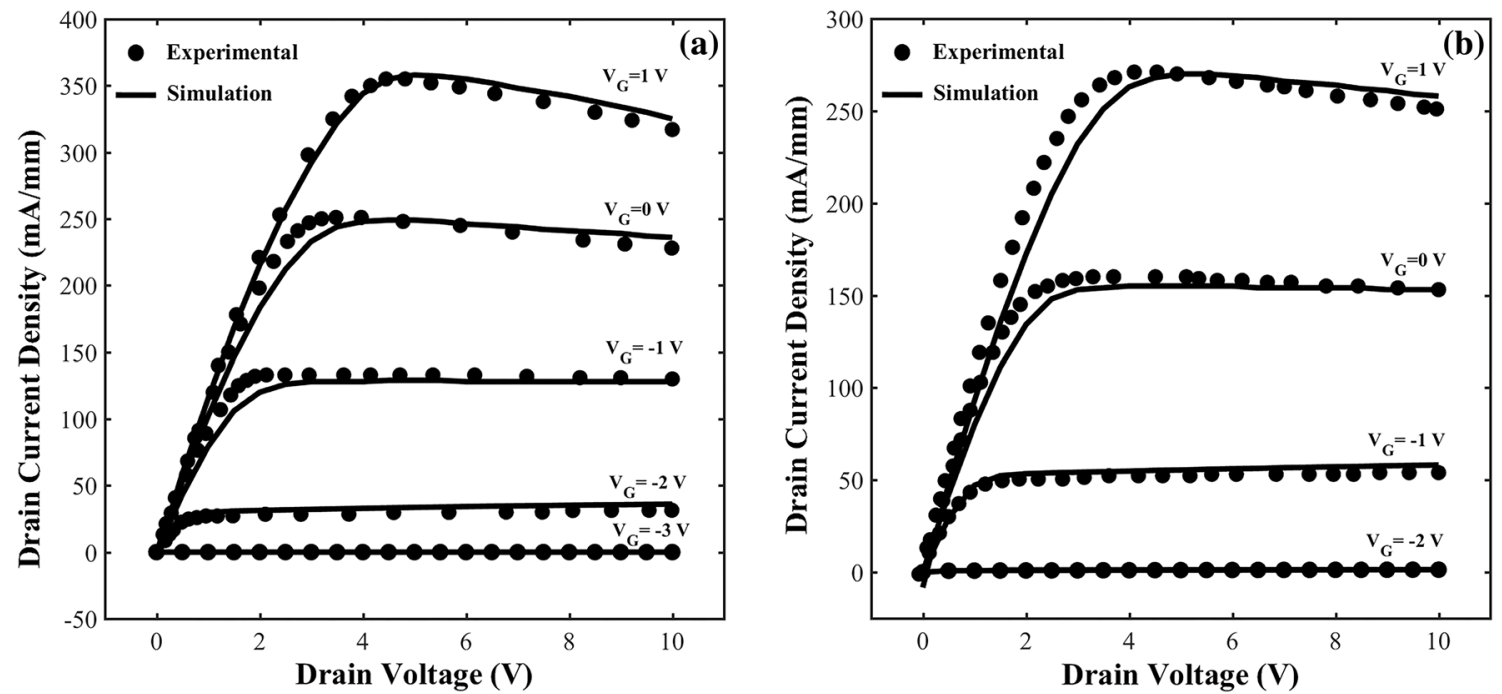

Fig. $3 I_{\mathrm{D}}-V_{\mathrm{D}}$ characteristics of (a) non-recessed and (b) 3-nm recessed-gate GaN HEMT. (Experimental data from Ref. 10). 
Table II Comparison of experimental data from literature ${ }^{10}$ and simulation results for GaN HEMT devices with non-recessed and 3-nm recessedgate structure; $n_{\mathrm{s}}$ calculated for equilibrium condition, and $I_{\mathrm{D}-\max }$ calculated at $V_{\mathrm{G}}=2 \mathrm{~V}$.

\begin{tabular}{|c|c|c|c|c|c|c|c|c|}
\hline \multirow[t]{2}{*}{ Sample } & \multicolumn{2}{|c|}{$V_{\text {th }}(\mathrm{V})$} & \multicolumn{2}{|l|}{$I_{\mathrm{D}-\max }(\mathrm{mA} / \mathrm{mm})$} & \multicolumn{2}{|c|}{$g_{\mathrm{m}-\max }(\mathrm{mS} / \mathrm{mm})$} & \multicolumn{2}{|c|}{$n_{\mathrm{s}}\left(\mathrm{cm}^{-2}\right)$} \\
\hline & Sim. & Exp. & Sim. & Exp. & Sim. & Exp. & Sim. & Exp. \\
\hline Non-recessed & -2.3 & -2.3 & $444\left(\right.$ at $\left.V_{\mathrm{G}}-V_{\mathrm{th}}=4.3 \mathrm{~V}\right)$ & 430 & 124 & 120 & $1 \times 10^{13}$ & $0.9 \times 10^{13}$ \\
\hline Recessed & -1.6 & -1.6 & $372\left(\right.$ at $\left.V_{\mathrm{G}}-V_{\mathrm{th}}=3.6 \mathrm{~V}\right)$ & 367 & 119 & 111 & $0.96 \times 10^{13}$ & - \\
\hline
\end{tabular}

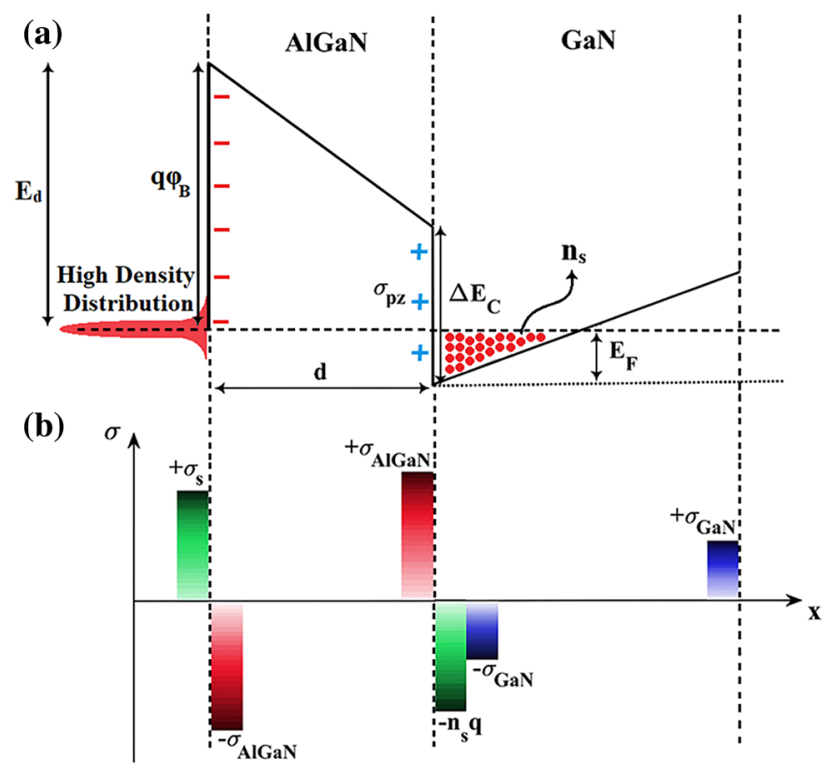

Fig. 4 (a) Energy-band diagram of AlGaN/GaN heterostructure. (b) Charge distribution at different interfaces, and formation of 2DEG with electrons supplied by surface donor states.

curves and equal parameters for the non-recessed and recessed-gate GaN HEMT structures confirm that the applied model exactly describes the physical phenomenon for both structures.

\section{Results and Discussion}

\section{Model Description for Non-recessed GaN HEMT}

A physics-based analytical model for the non-recessed gate GaN HEMT is presented to calculate the 2DEG charge concentration depending on the $\mathrm{AlGaN}$ barrier thickness.

Figure $4 \mathrm{a}$ depicts the band diagram of the GaN HEMT, and Fig. 4b shows the charge distribution profile, illustrating the formation of the 2DEG with electrons supplied by surface donor states. It is assumed that a high density of surface donor states exists at an energy level of $E_{\mathrm{d}}$ in the forbidden gap, measured relative to the conduction-band minimum of the AlGaN surface.
This model is based on the charge-neutrality condition across the barrier layer, where the unintentional doping of $\mathrm{GaN}$ and $\mathrm{AlGaN}$ is assumed to be negligible. Polarization in combination with the distributed surface donor states is the mechanism responsible for the 2DEG charge. By considering Fig. 4 , the 2 DEG charge concentration $\left(q n_{\mathrm{s}}\right)$ can be obtained by maintaining charge neutrality

$q n_{\mathrm{s}}=\frac{\varepsilon_{\mathrm{AlGaN}}}{d}\left[\frac{\sigma_{\mathrm{AlGaN}} d}{\varepsilon_{\mathrm{AlGaN}}}-\varphi_{\mathrm{b}}-\frac{E_{\mathrm{F}}}{q}+\frac{\Delta E_{\mathrm{C}}}{q}\right]$,

where $E_{\mathrm{F}}$ is the difference between the Fermi level and the conduction-band minimum (CBM) in the $\mathrm{GaN}$ at the heterointerface, $d$ is the AlGaN barrier thickness, and $\varepsilon_{\mathrm{AlGaN}}$ is the permittivity of the barrier layer. ${ }^{20}$

$\varepsilon_{\mathrm{AlGaN}}=10.4-0.3 x$

and $\varphi_{\mathrm{b}}$ is the Schottky barrier height, which can be calculated by assuming a constant surface donor state on the AlGaN surface, $n_{0}$, at a donor energy level $E_{\mathrm{d}}:^{18}$

$q \varphi_{\mathrm{b}}=\frac{n_{\mathrm{s}}}{n_{\mathrm{o}}}+E_{\mathrm{d}}$.

$E_{\mathrm{d}}$ and $n_{0}$ are linearly related to the $\mathrm{Al}$ mole fraction $(x)$ in the $\mathrm{AlGaN}$ barrier layer. ${ }^{19}$

$n_{0}=(2.9 x-0.0893) \times 10^{13} \mathrm{~cm}^{-2} \mathrm{eV}^{-1}$

$E_{\mathrm{d}}=2 x+0.42 \mathrm{eV}$

The energy band offset, $\Delta E_{\mathrm{C}}$, can be calculated from the bandgap energy of $\mathrm{AlGaN}$ and $\mathrm{GaN}$ as shown in the following equation, where the bandgap of $\mathrm{AlGaN}$ can be measured by $^{20}$

$\Delta E_{\mathrm{C}}=0.7\left(E_{\mathrm{g}}(x)-E_{\mathrm{g}}(0)\right)$

$E_{\mathrm{g}}(x)=x E_{\mathrm{g}}(\mathrm{AlN})+(1-x) E_{\mathrm{g}}(\mathrm{GaN})-x(1-x) \mathrm{eV}$

The polarization charge density $\left(\sigma_{\mathrm{AlGaN}}\right)$ is modeled by using spontaneous and piezoelectric polarization coefficients, which are functions of the mole fraction, AlGaN thickness, and lattice parameters of the GaN wurtzite crystal structure. 
Table III Mole fraction-dependent parameters of AlGaN/GaN heterostructure; data from Ref. 21.

\begin{tabular}{lcc}
\hline Symbol & Quantity & Expression \\
\hline$a$ & Lattice constant & $(-0.077 x+3.189) \times 10^{-10} \mathrm{~m}$ \\
$e_{31}$ & Piezoelectric coefficient & $(-0.11 x-0.49) \mathrm{C} / \mathrm{m}^{2}$ \\
$e_{33}$ & Piezoelectric coefficient & $(0.73 x+0.73) \mathrm{C} / \mathrm{m}^{2}$ \\
$C_{13}$ & Elastic constant & $(5 x+103) \mathrm{GPa}$ \\
$C_{33}$ & Elastic constant & $(-32 x+405) \mathrm{GPa}$ \\
$P_{\text {sp }}$ & Spontaneous polarization- & $(-0.052 x-0.029) \mathrm{C} / \mathrm{m}^{2}$ \\
& induced sheet charge & \\
\hline
\end{tabular}

$\sigma(x)=\left|2 S_{\mathrm{r}} \frac{a(0)-a(x)}{a(x)}\left\{e_{31}(x)-e_{33}(x) \frac{C_{13}(x)}{C_{33}(x)}\right\}+P_{\mathrm{sp}}(x)-P_{\mathrm{sp}}(0)\right|$,

where $a$ is the equilibrium value of the lattice constant, $e_{31}$ and $e_{33}$ are piezoelectric coefficients, $C_{13}$ and $C_{33}$ are elastic constants, and $P_{\mathrm{sp}}$ is the spontaneous polarization-induced sheet charge. All these parameters are listed as functions of the Al mole fraction in the $\mathrm{Al}_{x} \mathrm{GaN}_{1-x}$ layer in Table III.

$S_{\mathrm{r}}$ is a term to account for the residual strain induced in the layer, which is calculated based on the equation ${ }^{23}$

$S_{\mathrm{r}}=S_{\mathrm{cr}} \exp \left(1-\frac{d}{d_{\mathrm{cr}}}\right)$

where $d_{\mathrm{cr}}=\varepsilon_{\mathrm{AlGaN}}\left(E_{\mathrm{d}}-\Delta E_{\mathrm{c}}\right) / q \sigma_{\mathrm{pz}}$ is the critical thickness for the onset of significant charge at the interface and $S_{c r}$ is the critical strain extracted from Ref. 23.

Figure 5 calculates the 2DEG charge density for the GaN HEMT with respect to the AlGaN barrier layer thickness and Al mole fraction in the barrier layer. At low barrier thickness, the piezoelectric polarization component is dominant. However, when $d$ is substantially larger than the critical thickness (here $20 \mathrm{~nm}$ ), only the spontaneous polarization remains. This demonstrates that the total strain-induced polarization is reduced by increasing the barrier layer thickness $d$. At thicknesses higher than $d_{\text {cr }}$ $\left(d \gg d_{\text {cr }}\right)$, due to the decrease in the residual strain, the piezoelectric polarization becomes zero and the $2 \mathrm{DEG}$ reaches an almost constant value. These modeling results match with the report by Bykhovski et al. ${ }^{24}$

Moreover, $\mathrm{AlGaN}$ has a smaller lattice constant than $\mathrm{GaN}$. So, by increasing the Al mole fraction in $\mathrm{AlGaN}$, the mismatch and strain effects on the piezoelectric and spontaneous polarization gradually increase. Hence, the 2DEG charge density at the $\mathrm{AlGaN}-\mathrm{GaN}$ interface increases. In addition to physical justifications, this model is verified by the quite satisfactory agreement with experimental data in Fig. 5.

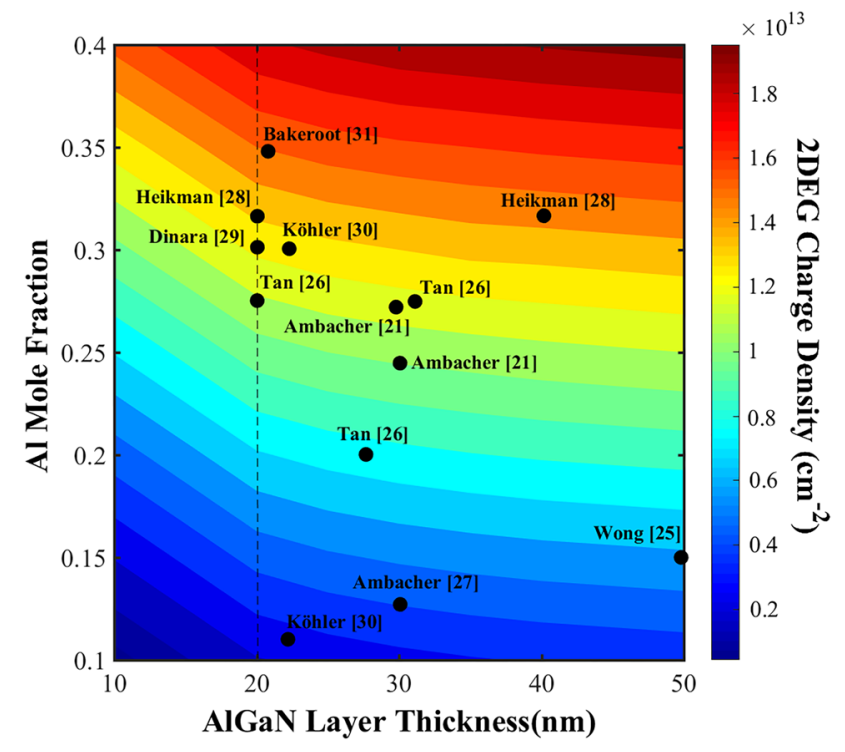

Fig. 5 Contour plot of 2DEG charge density for GaN HEMT as a function of barrier layer thickness $(d)$ and $\mathrm{Al}$ mole fraction in the barrier layer. Experimental data indicated by black dots..$^{21,25-31}$

\section{Model Description for Recessed-Gate GaN HEMT}

In recessed-gate structures, the barrier thickness under the gate decreases. Due to the constant electric field in the barrier layer, $E_{\mathrm{d}}$ moves away from the Fermi level and prevents the surface donor states from donating electrons to form 2DEG charge carriers at the AlGaN-GaN interface.

In recessed-gate GaN HEMT structures, to maintain charge neutrality, the electric field across the barrier layer is given below, where $h$ is the recess height in these structures (Fig. 1):

$\frac{\sigma_{\mathrm{AlGaN}}-q n_{\mathrm{s}}}{\varepsilon_{\mathrm{AlGaN}}}=\frac{q \varphi_{\mathrm{b}}+E_{\mathrm{F}}-\Delta E_{\mathrm{C}}}{q(d-h)}$.

The 2DEG electron density for the recessed-gate GaN HEMT structures can be calculated by applying Eqs. 1 and 3 to Eq. 10 and solving for the 2DEG charge density.

$q n_{\mathrm{s}}=\frac{\sigma_{\mathrm{AlGaN}} h+k_{1}}{h+k_{2}}$,

where

$k_{1}=\frac{\varepsilon_{\mathrm{AlGaN}}}{q}\left[E_{\mathrm{d}}+E_{\mathrm{F}}-\Delta E_{\mathrm{C}}\right]-d \sigma_{\mathrm{AlGaN}}$,

$k_{2}=-\left[d+\frac{\varepsilon_{\mathrm{AlGaN}}}{q^{2} n_{o}}\right]$

From Eq. 11 it can be seen that, by increasing the recess height, the $2 \mathrm{DEG}$ charge density will be decreased and the 
transistor starts to operate in E-mode. For a sufficient recess height to ensure that the GaN device demonstrates normallyOFF operation $(h \approx d)$, the critical 2DEG charge density can be calculated from Eqs. 11-13:

$n_{\mathrm{s}} \approx n_{\mathrm{o}}\left(E_{\mathrm{d}}+E_{\mathrm{f}}-\Delta E_{\mathrm{c}}\right)$.

Therefore, according to the proposed model, the $n_{\mathrm{s}}$ value for the experimental recessed-gate device with an $\mathrm{Al}$ mole fraction of 0.27 in the $\mathrm{AlGaN}$ barrier layer is calculated to be $0.48 \times 10^{13} \mathrm{~cm}^{-2}$. Figure 6 shows the 2DEG charge density modeled for the recessed-gate GaN HEMT structure with various $\mathrm{AlGaN}$ barrier thicknesses $(d)$.

In this figure, the critical recess height is indicated by a vertical dashed line, at which the charge density equals $n_{\mathrm{s}}=0.48 \times 10^{13} \mathrm{~cm}^{-2}$. Moreover, the accuracy of the current model is confirmed by the simulation results. Figure 7 shows the transfer characteristics of the $\mathrm{AlGaN} / \mathrm{GaN}$ HEMT for various recess heights with an AlGaN thickness of $d=15.6 \mathrm{~nm}$ and $\mathrm{Al}$ mole fraction of $x=0.27$.

This figure indicates that the critical recess height for this experimental device would be $h_{\mathrm{cr}} \approx 10 \mathrm{~nm}$, where $V_{\mathrm{th}}=0 \mathrm{~V}$ and the device will be normally-OFF. Comparison of Figs. 6 and 7 confirms that the simulation results are in good agreement with the analytical model.

The critical recess height in a GaN structure to convert it to a normally-OFF device depends on the $\mathrm{Al}$ mole fraction and the thickness of the barrier layer, as well as the energy level of donor states in the barrier layer. Since the recess

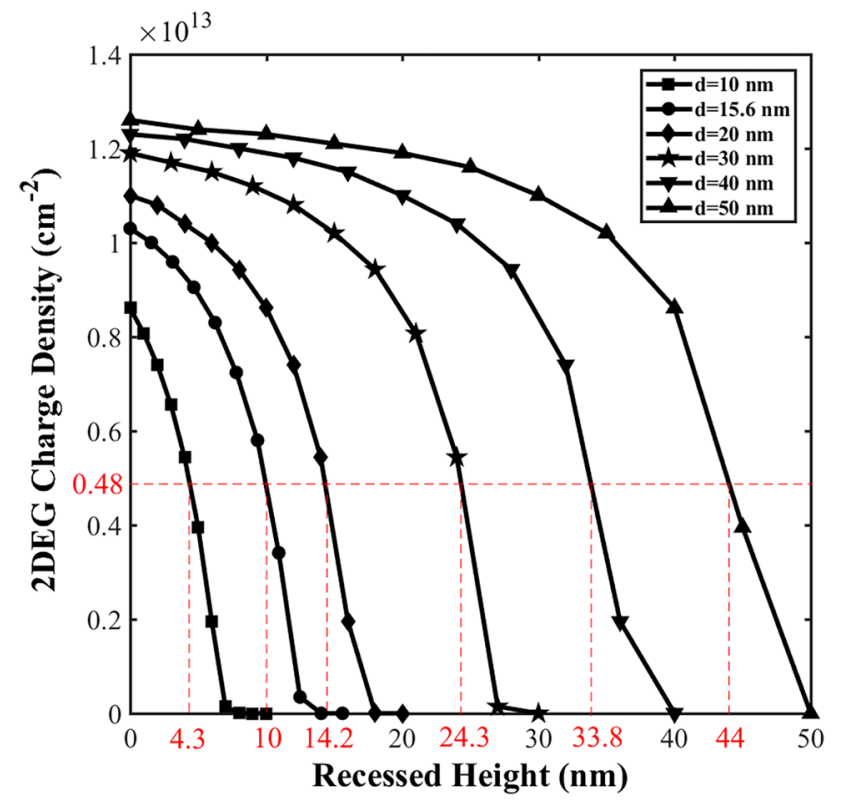

Fig. 6 The 2DEG charge density of the recessed-gate GaN HEMT structures versus the recess height for various barrier thicknesses. The critical recess height is determined by the vertical dashed line. height exceeds the critical recess height, the device converts to a normally-OFF $\mathrm{AlGaN} / \mathrm{GaN}$ device.

Figure 8 graphically illustrates the band energy for various recess heights $(h)$ and the resulting $2 \mathrm{DEG}$ charge density. The critical height $h=h_{\text {cr }}$ is the recess height at which $n_{\mathrm{s}}$ can be approximated to be very small, and the device can be considered as normally-OFF or in E-mode operation. This model shows how the 2DEG charge density can be controlled by varying the barrier thickness and recess height. It could help researchers develop new approaches for E-mode GaN HEMT technology.

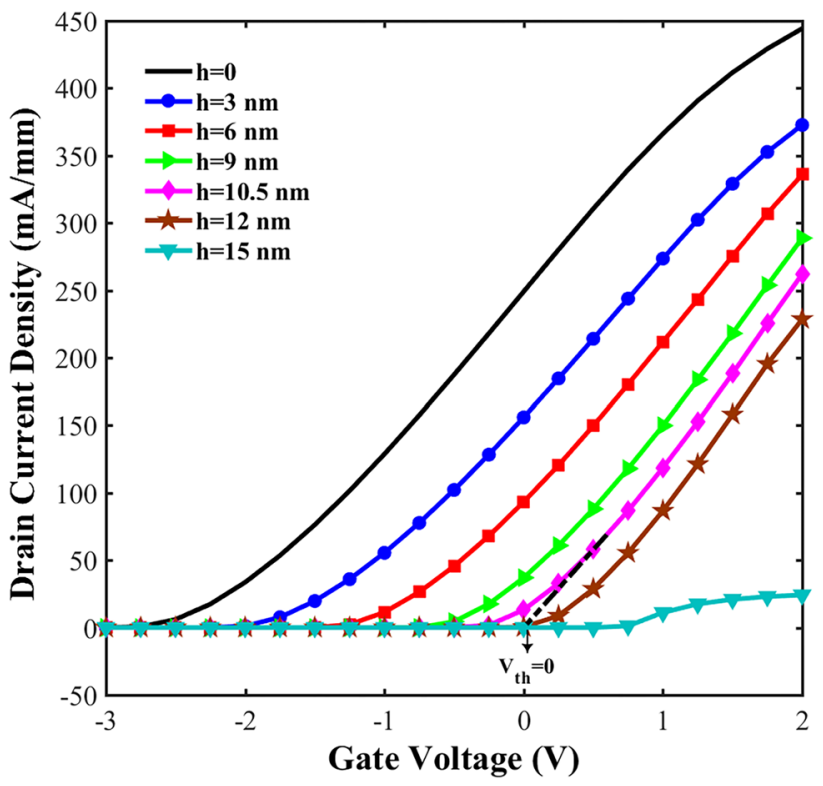

Fig. 7 Simulated DC transfer characteristics of AlGaN/GaN HEMT for various recess heights for AlGaN thickness $(d)$ of $15.6 \mathrm{~nm}$ and $\mathrm{Al}$ mole fraction $(x)$ of 0.27 .

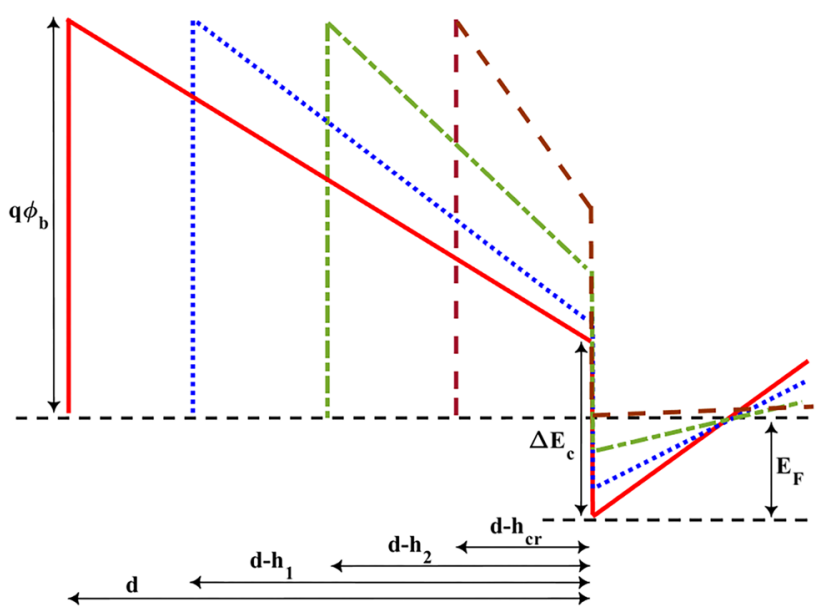

Fig. 8 Band diagrams of AlGaN/GaN HEMT for various recess heights $\left(h_{\mathrm{cr}}>h_{2}>h_{1}\right)$. 


\section{Conclusions}

GaN HEMTs with non-recessed and recessed-gate structures are investigated, achieving excellent agreement between the simulation results and experimental measurements. Moreover, an analytical model is provided for the 2DEG charge density of the GaN HEMTs with both structures. The importance of this model is that the optimized AlGaN layers can be designed regarding the thickness, the $\mathrm{Al}$ mole fraction, and the recess height to control the 2DEG charge density. Achieving a high 2DEG density is a critical priority to design reliable low-resistivity high-performance $\mathrm{GaN}$ HEMTs.

Moreover, the model can predict the experimental observations for recessed-gate GaN HEMTs in an analytical manner, while considering the effects of the mole fraction and recess height of the $\mathrm{AlGaN}$ barrier layer. A relation is developed between the recess height and 2DEG charge density and can be used to extract the critical recess height at which the device converts to normally-OFF operation. The model shows excellent agreement with experimentally measured data.

Acknowledgments This work was supported by Interreg DeutschlandDanmark with funds from the European Regional Development Fund via the PE-Region Platform project (ref. 098-1.1-18). Find further information on Interreg Deutschland-Denmark on www.interreg5a.eu

Conflict of Interest The authors declare that they have no conflicts of interest.

Open Access This article is licensed under a Creative Commons Attribution 4.0 International License, which permits use, sharing, adaptation, distribution and reproduction in any medium or format, as long as you give appropriate credit to the original author(s) and the source, provide a link to the Creative Commons licence, and indicate if changes were made. The images or other third party material in this article are included in the article's Creative Commons licence, unless indicated otherwise in a credit line to the material. If material is not included in the article's Creative Commons licence and your intended use is not permitted by statutory regulation or exceeds the permitted use, you will need to obtain permission directly from the copyright holder. To view a copy of this licence, visit http://creativecommons.org/licenses/by/4.0/.

\section{References}

1. N.M. Shrestha, Y.Y. Wang, Y. Li, and E.Y. Chang, Vacuum 118, 59 (2015).

2. N.M. Shrestha, Y. Li, and E.Y. Chang, J. Comput. Electron. 15, 154 (2016).

3. S. Sharbati, T. Ebel, and W.-T. Franke, Microelectron. Reliab. 114, 113907 (2020).

4. X. Lyu, H. Li, Y. Abdullah, K. Wang, B. Hu, Z. Yang, J. Liu, J. Wang, L. Liu, and S. Bala, IEEE Trans. Power Electron. 35, 8926 (2020)

5. J. Zhu, L. Chen, J. Jiang, X. Lu, L. Yang, B. Hou, M. Liao, Y. Zhou, X. Ma, and Y. Hao, IEEE Electron. Device Lett. 39, 79 (2018).

6. T. Zine-eddine, H. Zahra, and M. Zitouni, J. Sci. Adv. Mater. Devices 4, 180 (2019).
7. Y. Dong, Z. Xie, D. Chen, H. Lu, R. Zhang, and Y. Zheng, Int. J. Numer. Model. Electron. Netw. Dev. Field. 32, e2482 (2019).

8. D.K. Panda and T.R. Lenka, Microsyst. Technol. (2019). https://doi. org/10.1007/s00542-019-04324-3.

9. C. Yang, J. Xiong, J. Wei, J. Wu, B. Zhang, and X. Luo, Adv. Condens. Matter Phys. 2015, 267680 (2015).

10. N.M. Shrestha, Y. Li, T. Suemitsu, and S. Samukawa, IEEE Trans. Electron Devices 66, 1694 (2019).

11. C. Wood, and D. Jena, Polarization Effects in Semiconductors, $1 \mathrm{st}$ ed., (New York: Springer, 2008).

12. M. Higashiwaki, S. Chowdhury, M.-S. Miao, B.L. Swenson, C.G.V.D. Walle, and U.K. Mishra, J. Appl. Phys. 108, 063719 (2010).

13. M.S. Miao, J.R. Weber, and C.G.V.D. Walle, J. Appl. Phys. 107, 123713 (2010).

14. L. Gordon, M.-S. Miao, S. Chowdhury, M. Higashiwaki, U.K. Mishra, and C.G. Van de Walle, J. Phys. D Appl. Phys. 43, 505501 (2010).

15. F. Hemmi, C. Thomas, Y.-C. Lai, A. Higo, Y. Watamura, S. Samukawa, T. Otsuji, and T. Suemitsu, Solid State Electron. 137, 1 (2017).

16. S. Samukawa, K. Sakamoto, and K. Ichiki, Jpn. J. Appl. Phys. 40, L779 (2001).

17. T. Ohno, D. Nakayama, T. Okada, and S. Samukawa, Results Phys. 8,169 (2018).

18. N. Goyal, B. Iñiguez, and T.A. Fjeldly, Appl. Phys. Lett. 101, 103505 (2012).

19. N. Goyal, B. Iniguez, and T.A. Fjeldly, AIP Conf. Proc. 1566, 393 (2013).

20. O. Ambacher, B. Foutz, J. Smart, J.R. Shealy, N.G. Weimann, K. Chu, M. Murphy, A.J. Sierakowski, W.J. Schaff, L.F. Eastman, R. Dimitrov, A. Mitchell, and M. Stutzmann, J. Appl. Phys. 87, 334 (2000).

21. O. Ambacher, J. Smart, J.R. Shealy, N.G. Weimann, K. Chu, M. Murphy, W.J. Schaff, L.F. Eastman, R. Dimitrov, L. Wittmer, M. Stutzmann, W. Rieger, and J. Hilsenbeck, J. Appl. Phys. 85, 3222 (1999).

22. SILVACO ${ }^{\circledR}$ (2016) ATLAS ${ }^{\mathrm{TM}}$ User’s Manual. https://dynamic.silva co.com/dynamicweb/jsp/downloads/DownloadManualsAction.do? req=silen-manuals\&nm $=$ atlas

23. N. Goyal, and T.A. Fjeldly, J. Appl. Phys. 113, 014505 (2013).

24. A.D. Bykhovski, B.L. Gelmont, and M.S. Shur, J. Appl. Phys. 81, 6332 (1997).

25. L.W. Wong, S.J. Cai, R. Li, K. Wang, H.W. Jiang, and M. Chen, Appl. Phys. Lett. 73, 1391 (1998).

26. G. Tan, and Y. Su, Mod. Phys. Lett. B 25, 1293 (2011).

27. O. Ambacher, J. Majewski, C. Miskys, A. Link, M. Hermann, M. Eickhoff, M. Stutzmann, F. Bernardini, V. Fiorentini, V. Tilak, B. Schaff, and L.F. Eastman, J. Phys. Condens. Matter. 14, 3399 (2002).

28. S. Heikman, S. Keller, Y. Wu, J.S. Speck, S.P. DenBaars, and U.K. Mishra, J. Appl. Phys. 93, 10114 (2003).

29. S.M. Dinara, S.K. Jana, S. Ghosh, P. Mukhopadhyay, R. Kumar, A. Chakraborty, S. Bhattacharya, and D. Biswas, AIP Adv. 5, 047136 (2015).

30. K. Köhler, S. Müller, R. Aidam, P. Waltereit, W. Pletschen, L. Kirste, H.P. Menner, W. Bronner, A. Leuther, R. Quay, M. Mikulla, O. Ambacher, R. Granzner, F. Schwierz, C. Buchheim, and R. Goldhahn, J. Appl. Phys. 107, 053711 (2010).

31. B. Bakeroot, S. You, T.-L. Wu, J. Hu, M.V. Hove, B.D. Jaeger, K. Geens, S. Stoffels, and S. Decoutere, J. Appl. Phys. 116, 134506 (2014).

Publisher's Note Springer Nature remains neutral with regard to jurisdictional claims in published maps and institutional affiliations. 